\title{
Second-line chemotherapy using taxane in patients with advanced gastric cancer who presented with severe peritoneal metastasis: A multicenter retrospective study
}

Hiroyuki Arai

St. Marianna University School of Medicine

Masahiro Kawahira

Shizuoka Cancer Center

Hirofumi Yasui

Shizuoka Cancer Center

Toshiki Masuishi

Aichi Cancer Center Hospital

Kei Muro

Aichi Cancer Center Hospital

Takako Eguchi Nakajima ( $\nabla$ tnakajima@marianna-u.ac.jp )

St. Marianna University School of Medicine

Research article

Keywords: gastric cancer, peritoneal metastasis, chemotherapy, massive ascites, inadequate oral intake

Posted Date: August 30th, 2019

DOI: https://doi.org/10.21203/rs.2.12810/v1

License: (a) (i) This work is licensed under a Creative Commons Attribution 4.0 International License. Read Full License

Version of Record: A version of this preprint was published at International Journal of Clinical Oncology on October 15th, 2020. See the published version at https://doi.org/10.1007/s10147-020-01802-x. 


\section{Abstract}

Background Individuals with advanced gastric cancer (AGC) who present with severe peritoneal metastasis (SPM) have poor prognosis, and the need to improve treatment for such condition and survival time is not met. Moreover, there are only few data about the second-line treatment for patients with such condition. Methods This retrospective study included patients receiving taxane-based second-line chemotherapy at three institutions in Japan between 2010 and 2016. Patients with AGC who present with SPM were included if they had massive ascites and/or inadequate oral intake requiring intravenous nutritional support. The efficacy and safety of the treatment were evaluated. Results In the present study, 43 (40\%) of 108 patients had an Eastern Cooperative Oncology Group Performance Status score $>2$, and the median serum albumin level of the patients was $3.3 \mathrm{~g} / \mathrm{mL}$. Ramucirumab was used in combination with paclitaxel in 21 patients. The median overall survival (OS) and progression-free survival (PFS) were 5.1 and 2.8 months, respectively. Inadequate oral intake was considered a negative prognostic factor of both OS and PFS in the multivariate analysis. Three treatmentrelated deaths were observed, which include those attributed to febrile neutropenia, gastrointestinal perforation, and pneumonitis. Common grade $>3$ adverse events were neutropenia (35\%), leukopenia (30\%), anemia (24\%), and anorexia (16\%). We observed febrile neutropenia in $8 \%$ and gastrointestinal perforation in $4 \%$ of patients, and such conditions were primarily observed in patients with inadequate oral intake. Conclusions Taxane-based second-line chemotherapy was effective and safe for patients with AGC who present with SPM. Attention must be provided when treating patients with inadequate oral intake as they are likely to have short prognosis and serious toxicities.

\section{Background}

Gastric cancer (GC) is the fifth most common cancer and the third leading cause of cancer-related deaths worldwide [1]. The standard chemotherapy for advanced GC (AGC) is the combination of fluoropyrimidine and platinum, with trastuzumab if the patient with HER-2 positive GC, in the first-line setting [2-4] and paclitaxel plus ramucirumab in the second-line setting [5]. Although these chemotherapy treatments have survival benefits, AGC is not curable, and best supportive care (BSC) is recommended in patients with generally poor condition who cannot tolerate chemotherapy.

Peritoneal metastasis (PM) is common in individuals with AGC, and it causes serious clinical complications, such as massive ascites, bowel obstruction, jaundice, and hydronephrosis [6-7]. These complications can rapidly worsen a patient's general condition, making them ineligible for chemotherapy. PM is associated with poor prognosis, and the survival time of patients with severe PM (SPM) who present with massive ascites and/or bowel obstruction is extremely short even with chemotherapy [8-11]. Unfortunately, most pivotal phase III trials have not included patients with AGC who present with SPM; hence, the standard first- and second-line chemotherapy for such patients has not been established to date [2-5, 12]. Some retrospective studies have shown the efficacy and safety of fluoropyrimidine-based regimen as first-line chemotherapy for patients with AGC who present with SPM $[9-10,13]$.

Second-line chemotherapy for AGC is provided more frequently in Japan than in other countries, as shown by a high proportion of patients (approximately 75\%-80\%) receiving second-line chemotherapy in several Japanese phase III trials $[2,4,12]$. Second-line chemotherapy is effective in prolonging the survival time of patients [14]. 
As shown in the RAINBOW trial, the combination therapy of paclitaxel and ramucirumab, which targets vascular endothelial growth factor receptor-2, has been established as a new standard second-line treatment for AGC [5]. In patients with mild to moderate PM, the administration of paclitaxel weekly is considered a promising treatment that results in longer progression-free survival (PFS) compared with the best available 5-FU regimen in the Japan Clinical Oncology Group (JCOG) 0407 trial [15]. However, to date, there are only few data about the use of second-line chemotherapy in patients with SPM. Therefore, this study aimed to investigate the efficacy and safety of taxane-based second-line chemotherapy for patients with AGC who present with SPM.

\section{Methods}

\section{Patients}

We retrospectively reviewed the records of patients with AGC who presented with SPM and received taxanebased second-line chemotherapy between July 2010 and June 2016 at three institutions in Japan. All patient data were extracted from a database at each center. In this study, SPM was defined as PM associated with massive ascites and/or inadequate oral intake. This definition was based on previous retrospective studies [910, 13] and randomized phase II/III trials (JCOG1108/WJOG7312G trial: UMIN000010949) that investigated the efficacy of 5-FU//Heucovorin (/LV) plus paclitaxel compared to 5-FU//LV in patients with AGC who presented with SPM. Inadequate oral intake was defined as the need for intravenous nutritional support. The degree of ascites was evaluated via computed tomography and was classified as follows: none, undetectable; mild, localized to the pelvic cavity or upper abdominal cavity; moderate, inconsistent with either mild or massive ascites; and massive, extending continuously between the pelvic cavity and upper abdominal cavity. The eligibility criteria for this study were as follows: (1) histologically proven adenocarcinoma of the stomach or gastroesophageal junction, (2) with SPM during the initiation of second-line chemotherapy, (3) absence of concomitant advanced malignant disease, (4) refractory or intolerance to fluoropyrimidine (and trastuzumab if a patient has HER-2 positive disease), and (5) receiving taxane-based second-line chemotherapy after disease progression during first-line chemotherapy or recurrence within 6 months after the last adjuvant chemotherapy dose. We excluded patients with a history of taxane treatment and/or those with serious complications, such as active infection, renal failure (serum creatinine level $>3.0 \mathrm{mg} / \mathrm{dL}$ ), and hepatic failure or obstructive jaundice (serum total bilirubin level $>2.0 \mathrm{mg} / \mathrm{dL}$ ). This study was approved by the institutional review board of each center. A written informed consent was obtained from all patients before treatment initiation.

\section{Assessments}

We compared the degree of ascites between baseline and during treatment and determined the best responses in ascites, which were as follows: complete response (CR), the ascites completely disappeared; partial response (PR), there was a decrease by at least one degree from baseline; stable disease (SD), there was no change from baseline; progressive disease, there was an increase by at least one degree from baseline; and not evaluated, it was impossible to evaluate because fluid was drained before assessment or because there are no available records of the assessment results. We defined the response rate and disease control rate in ascites as the proportion of patients with the best CR or PR and the best CR, PR, or SD, respectively, among patients with ascites at baseline. The improvement rate of oral intake was defined as the proportion of patients whose oral 
intake improved and who did not require nutritional support for at least 7 days among the patients who had inadequate oral intake at baseline.

\section{Statistical Analysis}

OS was defined as the time from treatment initiation to death from any cause, and PFS was defined as the time from treatment initiation to disease progression or death from any cause. Time to treatment failure (TTF) was defined as the time from the initiation of treatment to the last dose of second-line chemotherapy. Both OS and PFS were estimated using the Kaplan-Meier method. Prognostic factors were evaluated in the univariate and multivariate analyses using Cox proportional hazards models. Covariates with a $p$-value $<0.20$ in the univariate analysis were included in the multivariate analysis. Fisher's exact test was used for the test of independence between two categorical groups. All analyses were two-sided, and a $p$-value $<0.05$ was considered statistically significant. All statistical analyses were performed using StatView software version 5.0 (SAS Institute, Cary, NC, the USA).

\section{Results}

\section{Patients}

In total, 115 patients with AGC who presented with SPM received taxane-based second-line chemotherapy. Seven patients (four with serum total bilirubin level $>2.0 \mathrm{mg} / \mathrm{dL}$, two with a history of receiving taxane, and one with serious infection) were excluded; thus, 108 patients were finally included. The characteristics of the patients are shown in Table 1. Forty-three (40\%) patients had an Eastern Cooperative Oncology Group Performance Status (PS) score $>2$ (including one patient with a PS score of 3 ), and the median serum albumin level of the patients was 3.3 (range: $1.8-4.3) \mathrm{g} / \mathrm{mL}$. Thirty (34\%) patients already had SPM before the initiation of first-line treatment. The detailed information about the number of patients receiving chemotherapy is as follows: paclitaxel $(n=80)$, paclitaxel plus ramucirumab $(n=21)$, nanoparticle albumin-bound paclitaxel $(n=3)$, 5 -FU/Heucovorin plus paclitaxel $(n=3)$, and docetaxel $(n=1)$. The following characteristics are more commonly observed in patients receiving paclitaxel plus ramucirumab $(n=21)$ than in those receiving treatment without ramucirumab ( $n=87)$ : intestinal histological type (33\% vs 10\%), PS score of 0 or 1 (76\% vs $56 \%$ ), metastatic sites $>2$ ( $81 \%$ vs $44 \%)$, and adequate oral intake (71\% vs $44 \%)$. The details are shown in Additional file 1.

\section{Reasons for Discontinuation of Treatment}

The median TTF was 2.2 months. The reasons for treatment discontinuation were as follows: disease progression ( $n=90[83 \%])$, adverse events $(n=11[10 \%])$, patient refusal $(n=3[3 \%])$, and other reasons $(n=4$ [4\%]). The adverse events leading to treatment discontinuation were peripheral neuropathy ( $n=4$, including three grade 3 and one grade 2 cases), gastrointestinal perforation ( $n=2$, including one grade 5 and one grade 3 cases), pneumonitis ( $n=2$, including one grade 5 and one grade 3 cases), febrile neutropenia ( $n=1$, grade 5 case), fatigue ( $n=1$, grade 2 case), and allergic reaction ( $n=1$, grade 3 case). 


\section{Efficacy}

In total, 105 (97\%) patients had died. The median OS and PFS were 5.1 and 2.8 months, respectively (Figure 1). In the analysis according to the subtype of SPM, the median OS of patients with massive ascites only, inadequate oral intake only, and both were 6.0,4.4, and 3.2 months, respectively. The median PFS were 3.5, 2.5, and 2.1 months, respectively (Figure 1). The univariate analysis of OS identified two prognostic factors: presence of massive ascites and inadequate oral intake. The multivariate analysis showed that inadequate oral intake was the only independent prognostic factor (hazard ration $[\mathrm{HR}]=2.41 ; 95 \%$ confidence interval [Cl]: $1.47-3.97 ; p<0.01$ ) (Table 2). Similarly, in the analysis of PFS, inadequate oral intake was the only independent prognostic factor in the multivariate analysis ( $\mathrm{HR}=1.88 ; 95 \% \mathrm{Cl}: 1.15-3.08 ; p=0.01$ ) (Table 2 ).

The response rate and disease control rate in ascites were $27 \%(28 / 102)$ and $78 \%(80 / 102)$, respectively. The improvement rate of oral intake was $31 \%$ (17/55) (Table 3).

\section{Safety}

Data about toxicity are shown in Table 4. Three (3\%) treatment-related deaths were recorded, which include those attributed to febrile neutropenia in a patient receiving paclitaxel plus ramucirumab, gastrointestinal perforation in a patient receiving paclitaxel plus ramucirumab, and pneumonitis in a patient receiving paclitaxel. In all patients, common grade $>3$ adverse events were neutropenia $(n=38[35 \%])$, leukopenia $(n=32[30 \%])$, anemia $(n=26[24 \%])$, and anorexia $(n=16[15 \%])$. Febrile neutropenia occurred in nine $(8 \%)$ patients, which included six patients with a PS score of 2 and seven patients whose oral intake were inadequate, and gastrointestinal perforation occurred in four (4\%) patients, which included two patients with a PS score of 2 and three patients whose oral intake were inadequate. The details of each case are shown in Additional file 2. Both febrile neutropenia and gastrointestinal perforation were more commonly observed in patients with inadequate oral intake than in those with adequate oral intake (13\% [7/55] vs $4 \%$ [2/53] and $5 \%[3 / 55]$ vs $2 \%[1 / 53]$, respectively). Meanwhile, higher incidence of febrile neutropenia and gastrointestinal perforation was not observed in patients with massive ascites, compared to those without massive ascites (5\% [4/79] vs 17\% [5/29] and $4 \%[3 / 79]$ vs $3 \%[1 / 29]$, respectively). Also, both febrile neutropenia and gastrointestinal perforation were more commonly observed in patients receiving paclitaxel plus ramucirumab treatment than in those receiving ramucirumab $(14 \%$ [3/21] vs $7 \%$ [6/87] and $10 \%$ [2/21] vs $2 \%$ [2/87], respectively). In patients with massive ascites, the incidence of febrile neutropenia and gastrointestinal perforation were almost similar between patients receiving paclitaxel plus ramucirumab treatment and those receiving treatment without ramucirumab ( $6 \%$ [1/16] vs $5 \%$ [3/63], $p>0.99$ and $6 \%$ [1/16] vs $3 \%$ [2/63], $p=0.50$, respectively). Meanwhile, in patients with inadequate oral intake, the incidence of febrile neutropenia tended to be higher, and that of gastrointestinal perforation was significantly higher in patients receiving paclitaxel plus ramucirumab treatment than in those receiving treatment without ramucirumab (33\% [2/6] vs $10 \%$ [5/49], $p=0.16$ and $33 \%[2 / 6]$ vs $2 \%$ [1/49], $p=$ 0.03 , respectively) (Table 5). Gastrointestinal bleeding occurred only in two (2\%) patients, and both patients received treatment without ramucirumab.

\section{Post-discontinuation Therapy (PDT)}


In total, $28(26 \%)$ patients received PDT. The regimens of PDT were as follows: irinotecan plus cisplatin $(n=6)$, irinotecan $(n=6)$, FOLFOX $(n=6), 5-F U / /$ LV $(n=3)$, ramucirumab $(n=2), S-1$ plus oxaliplatin $(n=1), S-1$ plus cisplatin $(n=1)$, and others $(n=3)$.

\section{Discussion}

This study first investigated second-line treatment specifically for patients with AGC who presented with SPM. Herein, we reveal the efficacy and safety of taxane-based chemotherapy using real-world clinical data.

In contrast with previous clinical trials in the second-line setting, patients with SPM had a poorer general condition. The high proportion of patients with a poor PS score $(>2,40 \%)$ and low serum albumin level (with a median of $3.3 \mathrm{~g} / \mathrm{mL}$ ) had significant characteristics, which were presented in the current study. These characteristics are similar to those of patients with AGC who presented with SPM and who received first-line chemotherapy, as reported in another retrospective study. That is, $40 \%$ had a PS score $>2$, and the median serum albumin level was $3.1 \mathrm{~g} / \mathrm{mL}$ in the first-line setting [13]. This may lead to poor prognosis: median OS of 5.1 months and median PFS of 2.8 months. Although this result was worse than survival times in second-line chemotherapy for generally advanced GC, with a median OS of 7.7-9.6 months and median PFS of 3.6-4.4 months [5, 15-17], taxane-based second-line chemotherapy may have more survival benefits than BSC in patients with SPM, considering the reported median survival time of 2.4-3.8 months in patients with AGC receiving BSC [18-21]. Importantly, we showed the difference in prognosis between the subtypes of SPM. That is, longer OS and PFS were observed in the subtype with massive ascites alone compared with the other two subtypes. Similarly, the multivariate analysis showed that inadequate oral intake was the only adverse prognostic factor of both OS and PFS. These findings indicated that patients with inadequate oral intake may have more aggressive state of disease than others; therefore, we should be cautious in terms of treatment.

The incidence of common adverse events was almost comparable to that of the effects of paclitaxel-based treatment, as reported in the RAINBOW and WJOG4007 trials in the second-line setting $[5,16]$. However, of note, febrile neutropenia and gastrointestinal perforation were more commonly observed in patients receiving paclitaxel plus ramucirumab than in those in the RAINBOW trial. A higher incidence of febrile neutropenia was observed in patients receiving paclitaxel plus ramucirumab (14\%), and it was modestly higher in patients receiving taxane only $(7 \%)$ than in those in the RANBOW trial (3\% in the paclitaxel plus ramucirumab arm and $2 \%$ in the paclitaxel plus placebo arm). Similarly, gastrointestinal perforation was more commonly observed in patients receiving paclitaxel plus ramucirumab (10\%) than in those receiving taxane only (2\%) and in those in the RAINBOW trial ( $1 \%$ in the paclitaxel plus ramucirumab arm and $0 \%$ in the paclitaxel plus placebo arm).

Interestingly, the higher incidence of febrile neutropenia and gastrointestinal perforation after paclitaxel plus ramucirumab compared to after taxane only was observed in the subgroup with inadequate oral intake, but not in the subgroup with massive ascites. This may be caused by patient's poor systemic conditions, particularly in the subgroup with inadequate oral intake. Of note, approximately $33 \%$ of patients who have inadequate oral intake and who were receiving paclitaxel plus ramucirumab presented with gastrointestinal perforation. This finding indicated that gastrointestinal perforation during anti-angiogenesis treatment is significantly correlated to severe peritoneal metastasis involving in the intestinal tract stenosis. A previous report has shown that tumor infiltration in the intestinal tract and stenosis due to PM might be the risk factors of gastrointestinal perforation 
[22]. These findings can serve as a warning to observe caution when providing treatment with paclitaxel plus ramucirumab particularly in patients with inadequate oral intake due to SPM.

This study had some limitations. First, this was a retrospective study. Second, the limited sample size made it challenging to obtain a clear conclusion. Thus, further studies must be conducted to validate the actual efficacy and safety of second-line chemotherapy in patients with AGC who presented with SPM in prospective clinical trials.

\section{Conclusion}

Taxane-based second-line chemotherapy was effective and safe in patients with AGC who presented with SPM. Attention must be provided when treating patients with inadequate oral intake as they are likely to have poor prognosis and serious toxicities.

\section{Abbreviation}

AGC, advanced gastric cancer

GC, gastric cancer

SPM, severe peritoneal metastasis

BSC, best supportive care

OS, overall survival

PFS, progression-free survival

$\mathrm{PM}$, peritoneal metastasis

SPM, severe PM

JCOG, Japan Clinical Oncology Group

$\mathrm{CR}$, complete response

PR, partial response

SD, stable disease

TTF, time to treatment failure

PDT, post-discontinuation therapy

\section{Declarations}

Ethics approval and consent to participate: 
This retrospective study was approved by Institutional Review Board (Ethics Committee, St. Marianna University School of Medicine) in accordance with the Japanese Ethical Guidelines for Medical and Health Research Involving Human Subjects. Written informed consent was obtained from all patients before treatment initiation.

\section{Consent for publication:}

Not applicable.

\section{Availability of data and materials}

The data that support the findings of this study are not available publicly. However, the data are available from the corresponding author upon reasonable request.

\section{Competing interests}

The authors declare that they have no competing interests.

\section{Funding}

We had no financial supports for this study.

\section{Authors' contributions}

HA and TEN planned, designed, analyzed and drafted the manuscript. MK and TM made substantial contributions to data collection. HY and KM supported planning and drafting the manuscript. All authors read and approved the final manuscript.

\section{Acknowledgments}

The authors would like to thank Enago (www.enago.com) for the English language review.

\section{Conflict of interest}

TEN reports personal fees from Bristol Myers-Squibb, Sawai, Nippon Kayaku, Yakult, Taiho, and Eli Lilly, and research grant from Bristol Myers-Squibb, Nippon Kayaku, Yakult, Taiho, and Eli Lilly.

\section{References}

1. International Agency for Research on Cancer. GLOBOCAN 2012: cancer incidence and mortality worldwide. http://gco.iarc.fr/. Accessed 22 Sept 2019. 
2. Koizumi W, Narahara H, Hara T, Takagane A, Akiya T, Takagi M, et al. S-1 plus cisplatin versus S-1 alone for first-line treatment of advanced gastric cancer (SPIRITS trial): a phase III trial. Lancet Oncol. 2008;9:215-21.

3. Bang YJ, Van Cutsem E, Feyereislova A, Chung HC, Shen L, Sawaki A, et al. Trastuzumab in combination with chemotherapy versus chemotherapy alone for treatment of HER2-positive advanced gastric or gastro-oesophageal junction cancer (ToGA): a phase 3, open-label, randomised controlled trial. Lancet. 2010;376:687-97.

4. Yamada Y, Higuchi K, Nishikawa K, Gotoh M, Fuse N, Sugimoto N, et al. Phase III study comparing oxaliplatin plus S-1 with cisplatin plus S-1 in chemotherapy-naive patients with advanced gastric cancer. Ann Oncol. 2015;26:141-8.

5. Wilke H, Muro K, Van Cutsem E, Oh SC, Bodoky G, Shimada Y, et al. Ramucirumab plus paclitaxel versus placebo plus paclitaxel in patients with previously treated advanced gastric or gastro-oesophageal junction adenocarcinoma (RAINBOW): a double-blind, randomised phase 3 trial. Lancet Oncol. 2014;15:1224-35.

6. Fujimura T, Ishii K, Oyama K, Miyashita T, Kinami S, Ninomiya I, et al. A new scoring system for peritoneal metastasis in gastric cancer. Gastric Cancer. 2003;6:146-52.

7. Wei J, Wu ND, Liu BR. Regional but fatal: intraperitoneal metastasis in gastric cancer. World J Gastroenterol. 2016;22:7478-85.

8. Iwasa S, Goto M, Yasui H, Nishina T, Takahari D, Nakayama NS, et al. Multicenter feasibility study of combination therapy with fluorouracil, leucovorin and paclitaxel (FLTAX) for peritoneal disseminated gastric cancer with massive ascites or inadequate oral intake. Jpn J Clin Oncol. 2012;42:787-93.

9. Iwasa S, Nakajima TE, Nakamura K, Takashima A, Kato K, Hamaguchi T, et al. First-line fluorouracil-based chemotherapy for patients with severe peritoneal disseminated gastric cancer. Gastric Cancer. 2012;15:21-6.

10. Hara H, Kadowaki S, Asayama M, Ooki A, Yamada T, Yoshii T, et al. First-line bolus 5fluorouracil plus leucovorin for peritoneally disseminated gastric cancer with massive ascites or inadequate oral intake. Int J Clin Oncol. 2018;23:275-80.

11. Shitara K, Mizota A, Matsuo K, Sato Y, Kondo C, Takahari D, et al. Fluoropyrimidine plus cisplatin for patients with advanced or recurrent gastric cancer with peritoneal metastasis. 
Gastric Cancer. 2013;16:48-55.

12. Boku N, Yamamoto S, Fukuda H, Shirao K, Doi T, Sawaki A, et al. Fluorouracil versus combination of irinotecan plus cisplatin versus S-1 in metastatic gastric cancer: a randomised phase 3 study. Lancet Oncol. 2009;10:1063-9.

13. Arai H, Iwasa S, Boku N, Kawahira M, Yasui H, Masuishi T, et al. Flioropyrimidine with or without platinum as first-line chemotherapy in patients with advanced gastric cancer and severe peritoneal metastasis: a multicenter retrospective study. BMC Cancer. 2019;19:652.

14. Iizumi S, Takashima A, Sakamaki K, Morita S, Boku N. Survival impact of post-progression chemotherapy in advanced gastric cancer: systematic review and meta-analysis. Cancer Chemother Pharmacol. 2018;81:981-9.

15. Nishina T, Boku N, Gotoh M, Shimada Y, Hamamoto Y, Yasui H, et al. Randomized phase II study of second-line chemotherapy with the best available 5-fluorouracil regimen versus weekly administration of paclitaxel in far advanced gastric cancer with severe peritoneal metastases refractory to 5-fluorouracil-containing regimens (JCOG0407). Gastric Cancer. 2016;19:902-10.

16. Hironaka S, Ueda S, Yasui H, Nishina T, Tsuda M, Tsumura T, et al. Randomized, open-label, phase III study comparing irinotecan with paclitaxel in patients with advanced gastric cancer without severe peritoneal metastasis after failure of prior combination chemotherapy using fluoropyrimidine plus platinum: WJOG 4007 trial. J Clin Oncol. 2013;31:4438-44.

17. Shitara K, Takashima A, Fujitani K, Koeda K, Hara H, Nakayama N, et al. Nab-paclitaxel versus solvent-based paclitaxel in patients with previously treated advanced gastric cancer (ABSOLUTE): an open-label, randomised, non-inferiority, phase 3 trial. Lancet Gastroenterol Hepatol. 2017;2:277-87.

18. Ford HE, Marshall A, Bridgewater JA, Janowitz T, Coxon FY, Wadsley J, et al. Docetaxel versus active symptom control for refractory oesophagogastric adenocarcinoma (COUGAR02): an open-label, phase 3 randomised controlled trial. Lancet Oncol. 2014;15:78-86.

19. Fuchs CS, Tomasek J, Yong CJ, Dumitru F, Passalacqua R, Goswami C, et al. Ramucirumab monotherapy for previously treated advanced gastric or gastro-oesophageal junction adenocarcinoma (REGARD): an international, randomised, multicentre, placebo-controlled, phase 3 trial. Lancet. 2014;383:31-9. 
20. Kang JH, Lee SI, Lim DH, Park KW, Oh SY, Kwon HC et al. Salvage chemotherapy for pretreated gastric cancer: a randomized phase III trial comparing chemotherapy plus best supportive care with best supportive care alone. J Clin Oncol. 2012;30:1513-18.

21. Thuss-Patience PC, Kretzschmar A, Bichev D, Deist T, Hinke A, Breithaupt K, et al. Survival advantage for irinotecan versus best supportive care as second-line chemotherapy in gastric cancer--a randomised phase III study of the Arbeitsgemeinschaft Internistische Onkologie (AIO). Eur J Cancer. 2011;47:2306-14.

22. Matsumoto H, Kawazoe A, Shimada K, Fukuoka S, Kuboki Y, Bando H, et al. A retrospective study of the safety and efficacy of paclitaxel plus ramucirumab in patients with advanced or recurrent gastric cancer with ascites. BMC Cancer. 2018;18:120.

\section{Tables}

Table 1. Characteristics of the patients 


\begin{tabular}{|c|c|c|c|}
\hline \multicolumn{2}{|c|}{ Characteristics } & \multicolumn{2}{|c|}{$(\mathrm{N}=108)(\%$} \\
\hline Age & Median (range) & 63 & $(25-83)$ \\
\hline \multirow[t]{2}{*}{ Sex } & Male & 62 & (57) \\
\hline & Female & 46 & $(43)$ \\
\hline \multirow[t]{4}{*}{ ECOG PS } & 0 & 10 & (9) \\
\hline & 1 & 55 & (51) \\
\hline & 2 & 42 & (39) \\
\hline & 3 & 1 & (1) \\
\hline \multirow[t]{2}{*}{ Histology } & Intestinal & 16 & (15) \\
\hline & Diffuse & 92 & (85) \\
\hline \multirow[t]{2}{*}{ Primary tumor } & Presence & 66 & (61) \\
\hline & Absence & 42 & (39) \\
\hline \multirow[t]{2}{*}{ Disease status } & Advanced & 76 & (70) \\
\hline & Recurrent & 32 & (30) \\
\hline Number of & 1 (only PM) & 53 & (49) \\
\hline metastatic sites & $\geq 2$ & 55 & (51) \\
\hline \multirow[t]{4}{*}{ Amount of ascites } & None & 6 & (6) \\
\hline & Mild & 16 & (15) \\
\hline & Moderate & 7 & (6) \\
\hline & Massive & 79 & (73) \\
\hline \multirow[t]{2}{*}{ Oral intake } & Adequate & 53 & (49) \\
\hline & Inadequate & 55 & (51) \\
\hline \multirow[t]{4}{*}{ Subtype of SPM } & Only massive ascites & 53 & (49) \\
\hline & Only inadequate oral & 29 & $(27)$ \\
\hline & intake & & \\
\hline & Both & 26 & $(24)$ \\
\hline \multirow{2}{*}{$\begin{array}{l}\text { Presence of SPM at the initiation of 1st-line } \\
\text { treatment }\end{array}$} & Yes & 37 & (34) \\
\hline & No & 71 & (66) \\
\hline \multirow{2}{*}{ Serum albumin level (g/mL) } & Median (range) & 3.3 & $(1.8-$ \\
\hline & & & 4.3) \\
\hline Serum CRP level (mg/dL) & Median (range) & 1.1 & $(0.0-$ \\
\hline
\end{tabular}


Agents used during the

1st-line treatment
$108 \quad(100)$

Platinum

CDDP

$44 \quad(41)$

$22 \quad(20)$

PTX

$80 \quad(74)$

PTX+RAM

Nab-PTX

FLTAX

DTX
21 (19)

3 (3)

3 (3)

1 (1)

CDDP: cisplatin, CRP: C-reactive protein, DTX: docetaxel, ECOG PS: Eastern Cooperative Oncology Group performance status, Nab-PTX: nanoparticle albumin-bound paclitaxel, OHP: oxaliplatin, PM: peritoneal metastasis, PTX: paclitaxel, RAM: ramucirumab, SPM: severe peritoneal metastasis

Table 2. Univariate and multivariate analyses of the prognostic factors of OS and PFS $(N=108)$ 


\begin{tabular}{|c|c|c|c|c|c|c|c|c|c|c|c|c|c|}
\hline \multirow{3}{*}{\multicolumn{2}{|c|}{ Variables }} & \multicolumn{6}{|c|}{ OS } & \multicolumn{6}{|c|}{ PFS } \\
\hline & & \multicolumn{3}{|c|}{ Univariate analysis } & \multicolumn{3}{|c|}{ Multivariate analysis } & \multicolumn{3}{|c|}{ Univariate analysis } & \multicolumn{3}{|c|}{ Multivariate analysis } \\
\hline & & HR & $\begin{array}{l}95 \% \\
\mathrm{CI}]\end{array}$ & $\begin{array}{c}p- \\
\text { value }\end{array}$ & HR & $\begin{array}{l}95 \% \\
\mathrm{CI}]\end{array}$ & $\begin{array}{c}p- \\
\text { value }\end{array}$ & HR & $\begin{array}{l}95 \% \\
\mathrm{CI}]\end{array}$ & $\begin{array}{c}p- \\
\text { value }\end{array}$ & HR & $\begin{array}{l}95 \% \\
\mathrm{CI}]\end{array}$ & $\begin{array}{c}p- \\
\text { value }\end{array}$ \\
\hline Age & $\begin{array}{c}\geq 65 \\
\text { (vs. }<65 \text { ) }\end{array}$ & 0.96 & $\begin{array}{l}{[0.65-} \\
1.42]\end{array}$ & 0.85 & & & & 1.00 & $\begin{array}{l}{[0.68-} \\
1.47]\end{array}$ & 0.99 & & & \\
\hline Sex & $\begin{array}{l}\text { Female } \\
\text { (vs. male) }\end{array}$ & 1.21 & $\begin{array}{l}{[0.81-} \\
1.78]\end{array}$ & 0.35 & & & & 1.14 & $\begin{array}{l}{[0.77-} \\
1.70]\end{array}$ & 0.51 & & & \\
\hline ECOG PS & $\begin{array}{c}2-3 \\
\text { (vs. 0-1) }\end{array}$ & 1.29 & $\begin{array}{l}{[0.87-} \\
1.91]\end{array}$ & 0.20 & & & & 1.18 & $\begin{array}{l}{[0.80-} \\
1.75]\end{array}$ & 0.41 & & & \\
\hline Histology & $\begin{array}{c}\text { Diffuse } \\
\text { (vs. } \\
\text { intestinal) }\end{array}$ & 1.17 & $\begin{array}{l}{[0.66-} \\
2.06]\end{array}$ & 0.60 & & & & 1.03 & $\begin{array}{l}{[0.59-} \\
1.78]\end{array}$ & 0.92 & & & \\
\hline $\begin{array}{l}\text { Disease } \\
\text { status }\end{array}$ & $\begin{array}{l}\text { Recurrent } \\
\text { (vs. } \\
\text { advanced) }\end{array}$ & 0.83 & $\begin{array}{l}{[0.55-} \\
1.27]\end{array}$ & 0.40 & & & & 0.81 & $\begin{array}{l}{[0.53-} \\
1.24]\end{array}$ & 0.34 & & & \\
\hline $\begin{array}{l}\text { Number of } \\
\text { metastatic } \\
\text { sites }\end{array}$ & $\geq 2$ (vs. 1 ) & 0.92 & $\begin{array}{l}{[0.63-} \\
1.36]\end{array}$ & 0.68 & & & & 0.84 & $\begin{array}{l}{[0.57-} \\
1.24]\end{array}$ & 0.38 & & & \\
\hline $\begin{array}{l}\text { SPM at 1st- } \\
\text { line } \\
\text { treatment } \\
\text { start }\end{array}$ & $\begin{array}{l}\text { Presence } \\
\text { (vs. } \\
\text { absence) }\end{array}$ & 1.08 & $\begin{array}{l}{[0.71-} \\
1.63]\end{array}$ & 0.72 & & & & 1.19 & $\begin{array}{l}{[0.79-} \\
1.79]\end{array}$ & 0.40 & & & \\
\hline $\begin{array}{c}\text { Massive } \\
\text { ascites }\end{array}$ & $\begin{array}{l}\text { Presence } \\
\text { (vs. } \\
\text { absence) }\end{array}$ & 0.65 & $\begin{array}{l}{[0.42-} \\
1.02]\end{array}$ & 0.06 & 1.20 & $\begin{array}{l}{[0.71-} \\
2.06]\end{array}$ & 0.50 & 0.72 & $\begin{array}{l}{[0.46-} \\
1.12]\end{array}$ & 0.14 & 1.11 & $\begin{array}{l}{[0.64-} \\
1.90]\end{array}$ & 0.71 \\
\hline Oral intake & $\begin{array}{l}\text { Presence } \\
\text { (vs. } \\
\text { absence) }\end{array}$ & 2.18 & $\begin{array}{l}{[1.45-} \\
3.29]\end{array}$ & $<0.01$ & 2.41 & $\begin{array}{l}{[1.47-} \\
3.97]\end{array}$ & $<0.01$ & 1.79 & $\begin{array}{l}{[1.19-} \\
2.68]\end{array}$ & $<0.01$ & 1.88 & $\begin{array}{l}{[1.15-} \\
3.08]\end{array}$ & 0.01 \\
\hline $\begin{array}{l}\text { Serum } \\
\text { albumin } \\
\text { level }\end{array}$ & $\begin{array}{c}<3.3 \mathrm{~g} / \mathrm{mL} \\
\\
\text { (vs. } \geq 3.3 \\
\mathrm{~g} / \mathrm{mL} \text { ) } \\
\end{array}$ & 1.07 & $\begin{array}{l}{[0.73-} \\
1.58]\end{array}$ & 0.72 & & & & 1.07 & $\begin{array}{l}{[0.72-} \\
1.58]\end{array}$ & 0.74 & & & \\
\hline
\end{tabular}

CI: confidence interval, ECOG PS: Eastern Cooperative Oncology Group performance status, HR: hazard ratio, OS: overall survival, SPM: severe peritoneal metastasis, PFS: progression-free survival

Table 3. Response rate in individuals with ascites and improvement rate of oral intake 


\begin{tabular}{|c|c|c|}
\hline & \multicolumn{2}{|r|}{ Patients with ascites at baseline $(\mathrm{N}=102)$} \\
\hline & $\mathrm{N} \quad(\%)$ & \\
\hline Complete response & $11(11)$ & \multirow{5}{*}{$\begin{array}{l}\text { RR in individuals with ascites: } 27 \% \\
\text { DCR in individuals with ascites: } 78 \%\end{array}$} \\
\hline Partial response & 17 (17) & \\
\hline Stable response & $52 \quad(51)$ & \\
\hline Progressive disease & $2(2)$ & \\
\hline \multirow[t]{3}{*}{ Not evaluated } & $20(20)$ & \\
\hline & \multicolumn{2}{|c|}{ Patients with inadequate oral intake at baseline $(\mathrm{N}=55)$} \\
\hline & $\mathrm{N} \quad(\%)$ & \\
\hline Improvement & $17 \quad(31)$ & \multirow[t]{2}{*}{ Improvement rate of oral intake: $31 \%$} \\
\hline No improvement & 38 (69) & \\
\hline
\end{tabular}

DCR: disease control rate, $R R$ : response rate

Table 4. Adverse events 


\begin{tabular}{|c|c|c|c|c|c|c|c|}
\hline \multirow[b]{3}{*}{ Adverse Event } & \multicolumn{2}{|c|}{$\begin{array}{c}\text { All patients }(\mathrm{N}= \\
108)\end{array}$} & \multicolumn{2}{|c|}{ PTX+RAM $(\mathrm{N}=21)$} & \multicolumn{3}{|c|}{$\begin{array}{c}\text { Taxane only }(\mathrm{N}= \\
\text { 87) }\end{array}$} \\
\hline & All Gr. & Gr. 3-4 & All Gr. & Gr. 3-4 & All Gr. & \multicolumn{2}{|c|}{ Gr. 3-4 } \\
\hline & $\mathrm{N} \quad(\%)$ & $\mathrm{N} \quad(\%)$ & $\mathrm{N} \quad(\%)$ & $\mathrm{N} \quad(\%)$ & $\mathrm{N} \quad(\%)$ & $\mathrm{N}$ & $(\%)$ \\
\hline Leukopenia & $82 \quad(76)$ & $32 \quad(30)$ & $14 \quad(67)$ & $6 \quad(29)$ & $68 \quad(78)$ & 26 & (30) \\
\hline Neutropenia & $72 \quad(67)$ & $38 \quad(35)$ & $14 \quad(67)$ & $7 \quad$ (33) & $58 \quad(67)$ & 31 & (36) \\
\hline Anemia & $75 \quad(69)$ & $26 \quad(24)$ & 11 & 1 (5) & $64 \quad(74)$ & 25 & (29) \\
\hline Thrombocytopenia & 11 & $2(2)$ & $3 \quad(14)$ & 1 (5) & $8 \quad(9)$ & 1 & (1) \\
\hline $\begin{array}{l}\text { Febrile } \\
\text { neutropenia }\end{array}$ & $9 \quad(8)$ & 9 (8) & $3 \quad(14)$ & 3 (14) & $6 \quad(7)$ & 6 & (7) \\
\hline Anorexia & $57 \quad(53)$ & 16 (15) & $13 \quad(62)$ & $0 \quad(0)$ & $44 \quad(51)$ & 16 & (18) \\
\hline Nausea & $47 \quad(44)$ & $5 \quad(5)$ & $8 \quad(38)$ & $0 \quad(0)$ & $39 \quad(45)$ & 5 & (6) \\
\hline Vomiting & $29 \quad(27)$ & 3 (3) & $4 \quad(19)$ & $0 \quad(0)$ & $25 \quad(29)$ & 3 & (3) \\
\hline Diarrhea & $26 \quad(24)$ & 1 (1) & $5 \quad(24)$ & $0 \quad(0)$ & 21 & 1 & (1) \\
\hline Stomatitis & $8 \quad(7)$ & $0 \quad(0)$ & 1 (5) & $0 \quad(0)$ & $7 \quad(8)$ & 0 & $(0)$ \\
\hline Fatigue & $76 \quad(70)$ & $8 \quad(7)$ & $17 \quad(81)$ & $0 \quad(0)$ & $59 \quad(68)$ & 8 & (9) \\
\hline $\begin{array}{l}\text { Sensory } \\
\text { neuropathy }\end{array}$ & $44 \quad(41)$ & $6 \quad(6)$ & 10 & $0 \quad(0)$ & $34 \quad$ (39) & 6 & (7) \\
\hline $\begin{array}{l}\text { Gastrointestinal } \\
\text { Perforation }\end{array}$ & $4 \quad(4)$ & $3 \quad(3)$ & $2 \quad(10)$ & 2 (10) & $2 \quad(2)$ & 1 & (1) \\
\hline $\begin{array}{l}\text { Gastrointestinal } \\
\text { bleeding }\end{array}$ & $2 \quad(2)$ & $2 \quad(2)$ & $0 \quad(0)$ & $0 \quad(0)$ & $2 \quad(2)$ & 2 & $(2)$ \\
\hline TRD & & & & & & & \\
\hline
\end{tabular}

PTX: paclitaxel, RAM: ramucirumab, TRD: treatment-related death 
Table 5. Comparison of the incidence of febrile neutropenia and gastrointestinal perforation according to the subtype of SPM between patients who received PTX+RAM and taxane only

\begin{tabular}{|l|c|c|c|c|c|c|c|c|c|c|}
\hline & \multicolumn{3}{|c|}{ Febrile neutropenia } & \multicolumn{3}{c|}{ Gastrointestinal perforation } \\
\cline { 2 - 11 } & \multicolumn{2}{|c|}{ PTX+RAM } & \multicolumn{2}{|c|}{ Taxane only } & $\begin{array}{c}p \text { - } \\
\text { value }\end{array}$ & \multicolumn{2}{c|}{ PTX+RAM } & \multicolumn{2}{c|}{$\begin{array}{c}\text { Taxane } \\
\text { only }\end{array}$} & $\begin{array}{c}p \text { - } \\
\text { value }\end{array}$ \\
\hline $\begin{array}{l}\text { (a) Only massive } \\
\text { ascites }\end{array}$ & $7 \%$ & $(1 / 15)$ & $3 \%$ & $(1 / 38)$ & 0.49 & $0 \%$ & $(0 / 15)$ & $3 \%$ & $(1 / 38)$ & $\begin{array}{c}> \\
0.99\end{array}$ \\
\hline $\begin{array}{l}\text { (b) Only inadequate } \\
\text { oral intake }\end{array}$ & $40 \%$ & $(2 / 5)$ & $13 \%$ & $(3 / 24)$ & 0.19 & $20 \%$ & $(1 / 5)$ & $0 \%$ & $(0 / 24)$ & $0 / 17$ \\
\hline $\begin{array}{l}\text { (c) Both } \\
\text { (d) With massive } \\
\text { ascites }\end{array}$ & $6 \%$ & $(0 / 1)$ & $8 \%$ & $(2 / 25)$ & $>$ & $100 \%$ & $(1 / 1)$ & $4 \%$ & $(1 / 25)$ & 0.08 \\
\hline $\begin{array}{l}\text { (e) Without massive } \\
\text { ascites }\end{array}$ & $40 \%$ & $(2 / 5)$ & $13 \%$ & $(3 / 24)$ & 0.19 & $20 \%$ & $(1 / 5)$ & $0 \%$ & $(0 / 24)$ & 0.17 \\
\hline $\begin{array}{l}\text { (f) Adequate oral } \\
\text { intake }\end{array}$ & $7 \%$ & $(1 / 15)$ & $3 \%$ & $(1 / 38)$ & 0.49 & $0 \%$ & $(0 / 15)$ & $3 \%$ & $(1 / 38)$ & $>$ \\
\hline $\begin{array}{l}\text { (g) Inadequate oral } \\
\text { intake }\end{array}$ & $33 \%$ & $(2 / 6)$ & $10 \%$ & $(5 / 49)$ & 0.16 & $33 \%$ & $(2 / 6)$ & $2 \%$ & $(1 / 49)$ & 0.03 \\
\hline
\end{tabular}

PTX: paclitaxel, RAM: ramucirumab, SPM: severe peritoneal metastasis

Patients included in the (d) subgroup consist of (a) and (c).

Patients included in the (e) subgroup are equal to (b).

Patients included in the (f) subgroup are equal to (a).

Patients included in the (g) subgroup consist of (b) and (c).

\section{Figures}


A

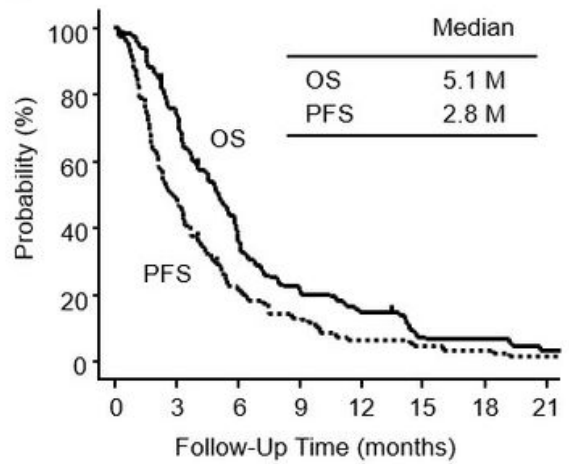

C

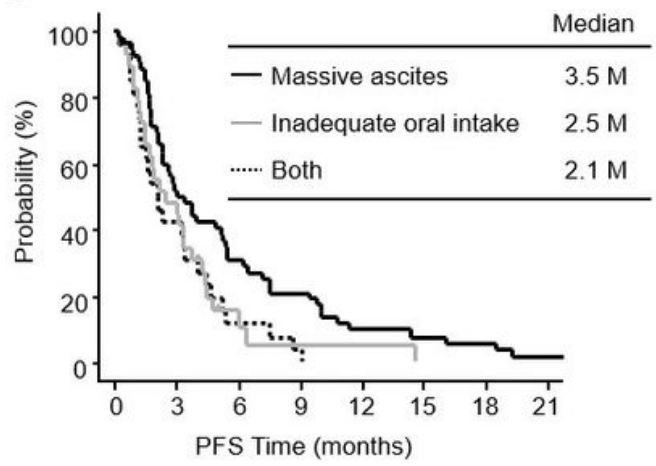

B

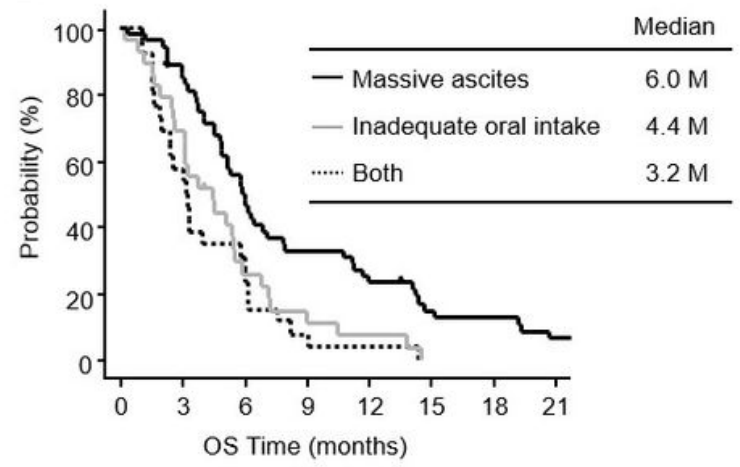

Figure 1

Kaplan-Meier analysis of overall survival (OS) and progression-free survival (PFS) A: Overall survival and progression-free survival of all patients $(\mathrm{N}=108)$ B: Overall survival of patients with massive ascites only $(\mathrm{N}=$ 53), inadequate oral intake only $(\mathrm{N}=29)$, and both $(\mathrm{N}=26) \mathrm{C}$ : Progression-free survival of patients with massive ascites only $(N=53)$, inadequate oral intake only $(N=29)$, and both $(N=26)$

\section{Supplementary Files}

This is a list of supplementary files associated with this preprint. Click to download.

- Additionalfile1.pptx

- Additionalfile2.pptx 